\title{
Résistance au cisaillement sous faible consolidation et structuration des argiles marines
}

\author{
Shear strength under low consolidation and structuration \\ of marine clays
}

\section{B. BENMOKRANE*, G. BALLIVY*, G. LEFEBVRE*, J.-P. LEBIHAN* et J. LOCAT**}

Rev. Franç. Géotech. n 64, pp. $31-43$ (juillet 1993)

\section{Résumé}

La mesure de la résistance au cisaillement d'argiles intactes en place ou en laboratoire est parfois impossible, que ce soit à cause des limitations des équipements disponibles ou de la difficulté d'accéder au dépôt (fonds marins...). II est encore important d'examiner si, à partir d'échantillons remaniés de ces mêmes dépôts, il est possible de recueillir des données propres au dépôt. II est proposé ici une méthode d'analyse en laboratoire à partir d'échantillons remaniés. Ainsi, des dépôts d'argile marine, mers de Tyrrell et de Champlain (Québec), maintenant émergés mais relativement jeunes (6 000 à 12000 ans) ont été étudiés sur le terrain puis en laboratoire avec des échantillons non remaniés. Puis les échantillons furent totalement remaniés et consolidés en laboratoire sous de faibles contraintes ( 7 à $150 \mathrm{kPa}$ ) ; ces échantillons furent ensuite soumis à des essais de pénétration au cône suédois. On a observé que l'évolution de $c_{u}$ (résistance au cisaillement non drainée) en fonction de $\sigma_{v}^{\prime}$ (contrainte effective de consolidation) se décompose en trois zones distinctes analogues aux zones observées sur les dépôts d'échantillons intacts ou sur le chantier. Ces résultats montrent que, au moins pour le cas des argiles de l'Est du Canada, caractérisées par une faible dégradation de la minéralogie de la roche mère, il est possible de prévoir le comportement des dépôts d'argile en place à partir d'essais de laboratoire sur des échantillons remaniés.

Mots-clés : argile intacte, argile remaniée, résistance au cisaillement non drainée, consolidation, thixotropie, structuration, dépôt d'argile.

\footnotetext{
Abstract

Shear strength evaluation of intact marine clay under deep waters is generally very difficult to assess. This can result from limitations of the in situ testing equipment (Shear vane) to collect undisturbed samples. It is examined here the possibility that remoulded samples collected from the marine floor can be helpful to obtain good correlations with the in-situ shear strength. Recent clay deposits presently above sea level have been tested on site and in the laboratory. Mechanical characterization have been conducted on intact samples. Subsequently they have been strongly remoulded and consolidated under very low stresses ( 7 to $150 \mathrm{kPa}$ ) equivalent to the actual vertical stresses in the natural deposit. The results show that shear strength variation of the consolidated remoulded samples follow the same evolution as the on-site intact clay i.e., overconsolidated behavior for low vertical stresses, then unconsolidated behavior and finally a normally consolidated state. The shear strength being proportional to the consolidation pressure. These results show that this type of clay remember the path stress imposed to the deposit and remoulding process is only partial. The testing of remoulded samples appear very important in this context.

Key words : intact clay, remoulded clay, undrained shear strength, consolidation, thixotropy, structuration, clay deposit.
} \footnotetext{
Département de génie civil, Université de Sherbrooke, Sherbrooke, Québec, Canada JIK 2R1, Tél. : (819) $821-7758$ ou 7114 ou
7115, Fax: (819) 821-7974.

** Département de géologie, Université Laval, Ste-Foy, Québec, Canada G1K 7P4
} 


\section{INTRODUCTION}

L'étude du développement de la résistance au cisaillement des argiles d'âge récent dans les fonds marins ou lacustres actuels est d'un grand intérêt pour les études d'aménagement off-shore ou pour la compréhension des dépôts émergés formés récemment, soit dans les 10000 ou 20000 dernières années. Les techniques d'investigation off-shore sont complexes et souvent fort coûteuses: le prélèvement d'échantillons non remaniés est particulièrement difficile. Dans une étape d'investigation préliminaire, il est proposé ici de définir le profil de résistance au cisaillement de ces dépôts marins ou lacustres à partir de résultats obtenus en laboratoire avec des échantillons remaniés, donc d'un prélèvement beaucoup plus facile, particulièrement dans le cas de relevés en mer.

Les argiles étudiées ici sont d'origine marine ou saumâtre en dépôt pélagique. Elles appartiennent à des dépôts présentement émergés depuis environ 6000 ans (mer de Tyrrell, baie James) ou environ 12000 ans (mer de Champlain, au niveau de Trois-Rivières). Elles proviennent de la mer de Tyrrell (site de la rivière Broadback) et de la mer de Champlain (sites de SaintLéon et Saint-Marcel) situés au Québec (fig. 1) et ont déjà été l'objet de nombreuses études géotechniques (LEFEBVRE, 1981; MORISSETTE, 1983), Leurs caractéristiques géotechniques à l'état naturel sont illustrées dans le tableau I. La salinité de l'eau interstitielle varie entre $15 \mathrm{~g} / 1$ (Saint-Léon) et $0,45 \mathrm{~g} / 1$ (Broadback) pour les échantillons concernés.

\section{PROGRAMME EXPÉRIMENTAL}

\subsection{Mode de préparation de l'argile remaniée}

Le programme expérimental comporte une reconsolidation œedométrique d'échantillons d'argile ayant été soumis à un remaniement usuel défini ainsi : à partir d'un bloc d'argile intact, on découpe des tranches que l'on place dans un récipient de porcelaine (après avoir éliminé les zones extérieures affectées par l'enrobage). L'argile mise dans le récipient est ensuite triturée vigoureusement à la spatule jusqu'à l'obtention d'un remaniement complet, c'est-à-dire un mélange homogène de consistance stable. Une portion de cette argile remaniée est ensuite placée à la spatule dans un récipient, en trois couches successives en éliminant au maximum les bulles d'air, pour effectuer un essai de pénétration au cône suédois (GARNEAU et LEBIHAN, 1977). L'argile est ensuite retournée au récipient de porcelaine et triturée à nouveau vigoureusement. Suite à cette deuxième opération de remaniement, on procède à nouveau à un essai de pénétration. Si les deux essais de pénétration séparés par un nouveau remaniement different de plus de $2 \%$, l'argile est remaniée à nouveau. Cette opération est répétée tant que deux essais de pénétration successifs séparés par un nouveau remaniement ne different pas de plus de $2 \%$ de la valeur moyenne.
Tableau I. - Caractéristiques géotechniques des trois argiles marines étudiées. Etat naturel.

Table 1. - Geotechnical characteristics for the three marine clays under study. Undisturbed state.

\begin{tabular}{|c|c|c|c|}
\hline & \multirow{2}{*}{$\begin{array}{c}\begin{array}{c}\text { Argile } \\
\text { de la mer } \\
\text { Tyrrel }\end{array} \\
\begin{array}{c}\text { Site de } \\
\text { Broadback }\end{array} \\
\end{array}$} & \multicolumn{2}{|c|}{$\begin{array}{l}\text { Argile de la mer } \\
\text { de Champlain }\end{array}$} \\
\hline & & $\begin{array}{l}\text { Site de } \\
\text { St-Léon }\end{array}$ & $\begin{array}{l}\text { Site de } \\
\text { St-Marcel }\end{array}$ \\
\hline $\begin{array}{l}\text { Profondeur } \\
\text { de prélèvement } \\
\text { du bloc utilisé }(\mathrm{m})\end{array}$ & 5,90 & 5,35 & 10,50 \\
\hline $\begin{array}{l}\text { Teneur en eau naturelle, } \\
w_{\text {nat }}(\%)\end{array}$ & 46 & 48,5 & 80 \\
\hline $\begin{array}{l}\text { Limite de plasticité } w_{p} \\
(\%)\end{array}$ & 26,6 & 21.4 & 25 \\
\hline $\begin{array}{l}\text { Limite de liquidité, } \\
\text { au cône suédois, } \\
\mathrm{w}_{\mathrm{LC}}(\%)\end{array}$ & 49,5 & 53 & 62 \\
\hline $\begin{array}{l}\text { Indice de plasticité, } \\
I_{p}(\%)\end{array}$ & 22,9 & 31,6 & 37 \\
\hline Indice de liquidité, ILC & 0,85 & 0,86 & 1,49 \\
\hline $\begin{array}{l}\text { Salinité de l'eau } \\
\text { interstitielle, }(\mathrm{g} / \mathrm{l})\end{array}$ & 0,45 & 15 & 2 \\
\hline $\begin{array}{l}\text { Pourcentage } \\
\text { de la fraction inférieure } \\
\text { à } 2 \mu(\%)\end{array}$ & 75 & 65 & 75 \\
\hline $\begin{array}{l}\text { Surface spécifique, } \\
\left(\mathrm{m}^{2} / \mathrm{g}\right)\end{array}$ & 40 & 40 & 67 \\
\hline Phyllo-silicates (\%) & 7 & 22 & 25 \\
\hline
\end{tabular}

$\cdot \mathrm{I}_{\mathrm{LC}}=\left(\mathrm{w}_{\text {nat }}-\mathrm{w}_{\mathrm{p}}\right) / \mathrm{I}_{\mathrm{p}}$

\subsection{Essai de reconsolidation œdométrique et évaluation de la résistance au cisaillement non drainée}

Les essais de reconsolidation œedométrique ont été réa. lisés à l'aide d'une cellule de consolidation spéciale conçue lors de travaux antérieurs à l'Université de Sherbrooke (LOCAT and LEFEBVRE, 1983). Cette cellule est constituée d'un cylindre d'acrylique de $102 \mathrm{~mm}$ de diamètre tel qu'illustré à la figure 2 . L'application de la pression verticale est transmise à l'échantillon à l'aide d'un piston doté d'une pierre poreuse au contact avec le sol. L'évacuation de l'eau à la base du cylindre est contrôlée par une valve. Ainsi, le drainage peut être assuré aux deux extrémités ou uniquement à la face supérieure comme ce fut le cas pour les essais de consolidation effectués avec mesure de pression interstitielle. Parallèlement, la perméabilité est mesurée à laide d'une colonne d'eau à charge variable.

Afin d'obtenir une bonne mise en place de l'argile remaniée dans la cellule de consolidation, on a augmenté, lors de l'opération du remaniement de l'argile intacte, la teneur en eau naturelle qui est de $46 \%, 48,5 \%$ et $80 \%$ pour Broadback, Saint-Léon et Saint-Marcel, à des 


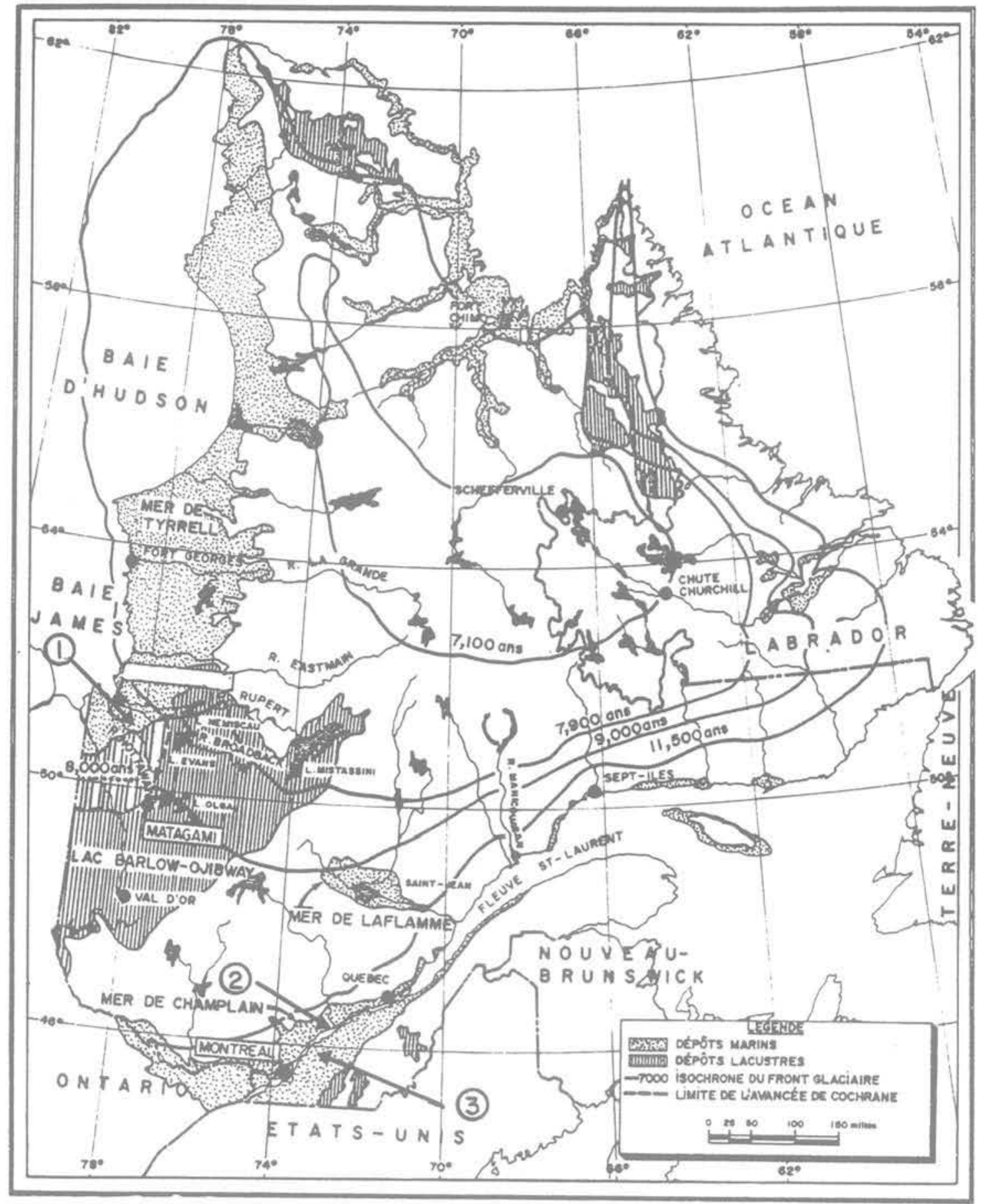

1. SITE DE BROADBACK (mer de Tyrrell)

2. SITE DE SAINT-LEON (mer de Champlain)

3. SITE DE SAINT-MARCEL (mer de Champlain)

Fig. 1. - Localisation des sites (BALLIVY et al., 1971).

Fig. 1. - Site location (BALLIVY et al., 1971). 


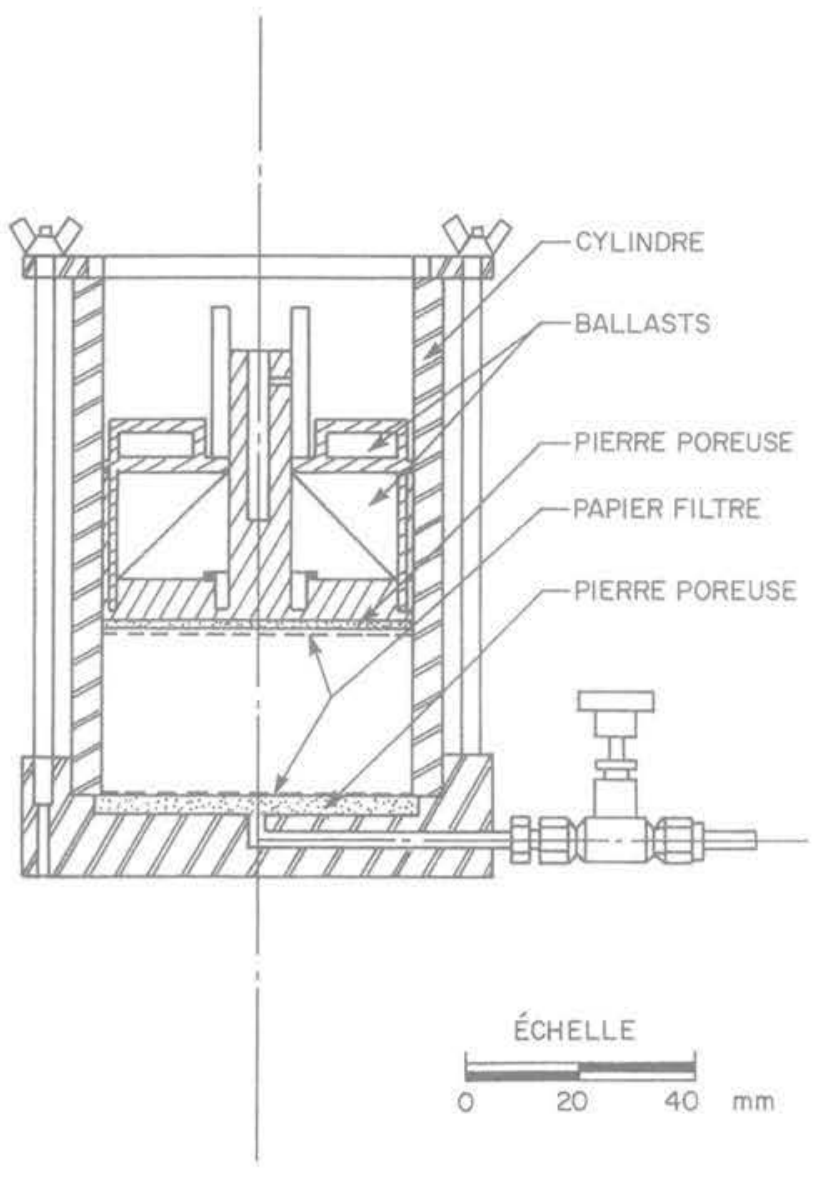

Fig. 2, - Cellule utilisée pour les essais de consolidation.

Fig. 2. - Cell used for the consolidation test.

valeurs respectives de mise en place de $52 \%, 62,5 \%$ et $85 \%$. Le tableau II donne un résumé des valeurs de différentes caractéristiques géotechniques des trois argiles lors de leur mise en place dans la cellule de consolidation. La hauteur initiale des échantillons, après leur mise en place dans la cellule, était d'environ $50 \mathrm{~mm}$. Il furent consolidés sous de faibles contraintes, variant de 7 à $95 \mathrm{kPa}$ et traduisant des conditions éventuelles dans des dépôts récents encore immergés. Ces êchantillons furent ensuite soumis à des essais de pénétration au cône suédois pour évaluer la résistance au cisaillement non drainée $c_{4}$ et ainsi permettre l'étude de la relation $c_{u}-\sigma_{v}^{\prime}\left(\sigma_{v}^{\prime} \stackrel{c^{u}}{=}\right.$ pression de consolidation).

Des essais ont été réalisés dans le but de pouvoir établir une comparaison entre le $c_{4}$ évalué au cône suédois et le $c_{\mathrm{u}}$ évalué en cisaillement simple à volume constant. Ainsi, des échantillons furent soumis à la mesure de $c_{u}$ au cône suédois, puis à des essais de cisaillement simple à volume constant après une période de reconsolidation aux mêmes pressions qu'ils avaient subies auparavant dans la cellule de consolidation.

\section{RÉSULTATS ET DISCUSSION}

\subsection{Variation de la résistance au cisaillement remaniée $\mathrm{c}_{\mathrm{u}}$ en fonction de l'indice de liquidité}

Les essais de pénétration au cône suédois effectués sur des échantillons remaniés, à teneur en eau naturelle et à teneur en eau augmentée, des trois argiles étudiées confirment que la résistance au cisaillement de l'argile remaniée, $\mathrm{c}_{\mathrm{u}_{\mathrm{r}}}$, est reliée par une relation unique à l'indice de liquidité, $I_{L C}$, au moins pour les argiles considérées de l'Est du Canada (LEROUEIL et al., 1983; LOCAT et LEFEBVRE, 1986). La relation proposée par LEROUEIL et al., est la suivante :

$$
c_{\mathrm{ur}}(\mathrm{kPa})=\frac{1}{\left(\mathrm{I}_{\mathrm{LC}}-0,21\right)^{2}}
$$

où $I_{L C}=$ indice de liquidité calculé en utilisant la valeur de la limite $\left(\mathrm{W}_{1}\right)$ déterminée au cône suédois (norme du Bureau de Normalisation du Québec 2501-092).

La relation (1) est illustrée à la figure 3 où on a reporté également les résultats obtenus dans le cadre de cette étude-ci.

\section{Tableau II. - Caractéristiques géotechniques des trois argiles étudiées lors de leur mise en place dans la cellule de consolidation.}

Table II. - Geotechnical characteristics for the three marine clays under study during their set up in the consolidation test cell.

\begin{tabular}{|l|c|c|c|c|c|c|c|}
\hline & $W_{\text {nat }}(\%)$ & $w_{\text {initial }}(\%)$ & $w_{p}(\%)$ & $w_{\text {LC }}(\%)$ & $I_{p}(\%)$ & $I_{\text {LCinitial }}$ & $e_{\text {initial }}$ \\
\hline Broadback & 46 & 52 & 26,60 & 47,50 & 20,90 & 1,21 & 1,423 \\
\hline Saint-Léon & 48,50 & 62,50 & 21,40 & 49,50 & 28,10 & 1,46 & 1,743 \\
\hline Saint-Marcel & 80 & 85 & 25 & 59 & 34 & 1,76 & 2,360 \\
\hline
\end{tabular}

- Initial: Valeur du paramètre lors de la mise en place de l'argile remaniée dans la cellule de consolidation.

$W_{\text {nat }}$ : Teneur en eau de l'argile à l'état naturel; $W_{\text {initial }}=$ teneur en eau initiale; $W_{p}:$ limite de plasticité; $W_{L C}$ : limite de

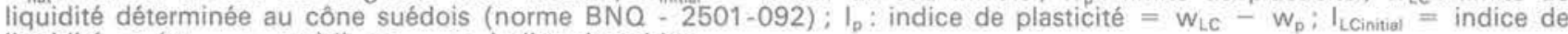
liquidité $=\left(w_{\text {initial }}-w_{p}\right) / I_{p} ; e_{\text {initial }}=$ indice des vides. 


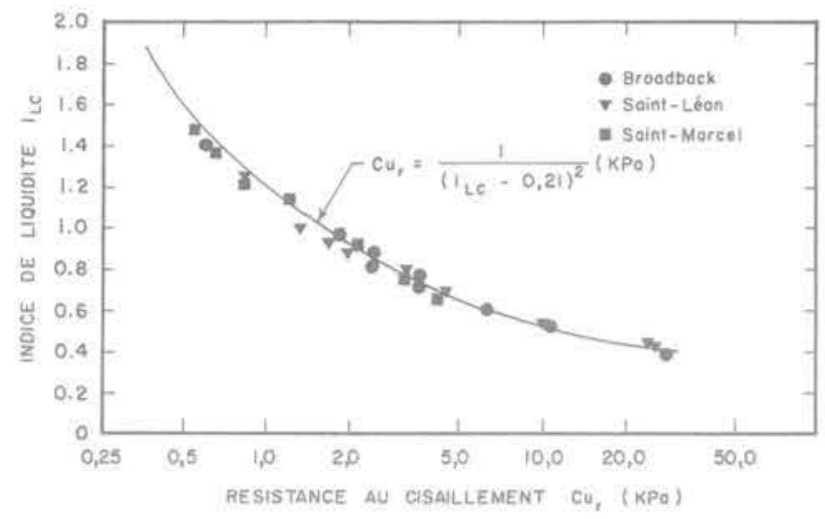

Fig. 3. - Variation de la résistance au cisaillement d'argile remaniée en fonction de l'indice de liquidité.

Fig. 3. - Relationship between the shear strength and liquidity index for remoulded clay.

\subsection{Gain thixotropique}

La thixotropie est un processus d'augmentation de résistance et ce à volume constant. Le gain de résistance résulterait d'une réorientation des molécules d'eau au pourtour des particules (MESRI et al., 1975). Ainsi, à la suite du remaniement d'une argile, on observe, à partir d'une valeur minimale évaluée immédiatement après, un gain de résistance à volume constant, et ce phénomène est désigné par un gain thixotropique.

Une partie de largile remaniée préparée initialement pour les essais de consolidation fut gardée dans un contenant fermé en plastique et conservé dans une chambre humide (humidité relative $=100 \%$, tempé. rature $=8^{\circ} \mathrm{C}$ ). Des mesures de résistance au cône suédois furent effectuées dans le temps, durant deux mois, afin d'évaluer les gains de résistance dus à la thixotropie. A la fin de ces deux mois, largile fut remaniée, et les mêmes opérations furent réalisées pendant un autre cycle d'une durée de deux mois.

Les résultats de ces essais effectués sur les trois argiles étudiées sont présentés à la figure 4. Ces résultats conduisent aux observations suivantes:

- l'évolution de la résistance en fonction du temps est identique pour les deux cycles d'essais réalisés (durée d'un cycle $=2$ mois). Ceci indique qu'un deuxième remaniement complet de largile n'affecte pratiquement pas les gains thixotropiques;

- pour la durée d'entreposage considérée ici, la courbe du gain thixotropique en fonction du temps, pour chaque type d'argile, est constituée de deux phases. Une première phase, d'une durée allant jusqu'à un mois, dans laquelle on assiste à une forte augmentation de la résistance, puis une deuxième phase caractérisée par une augmentation de résistance lente et uniforme;

- à une durée égale de conservation, les valeurs de gain de résistance pour largile de Broadback sont supérieures à celles des argiles de Saint-Léon et de SaintMarcel. Les valeurs relevées après deux mois sont de $6,1,3,5$ et $4,5 \mathrm{kPa}$ respectivement pour Broadback, Saint-Léon et Saint-Marcel, alors que c après remaniement est de l'ordre de $0,5 \mathrm{kPa}$ pour les trois argiles.
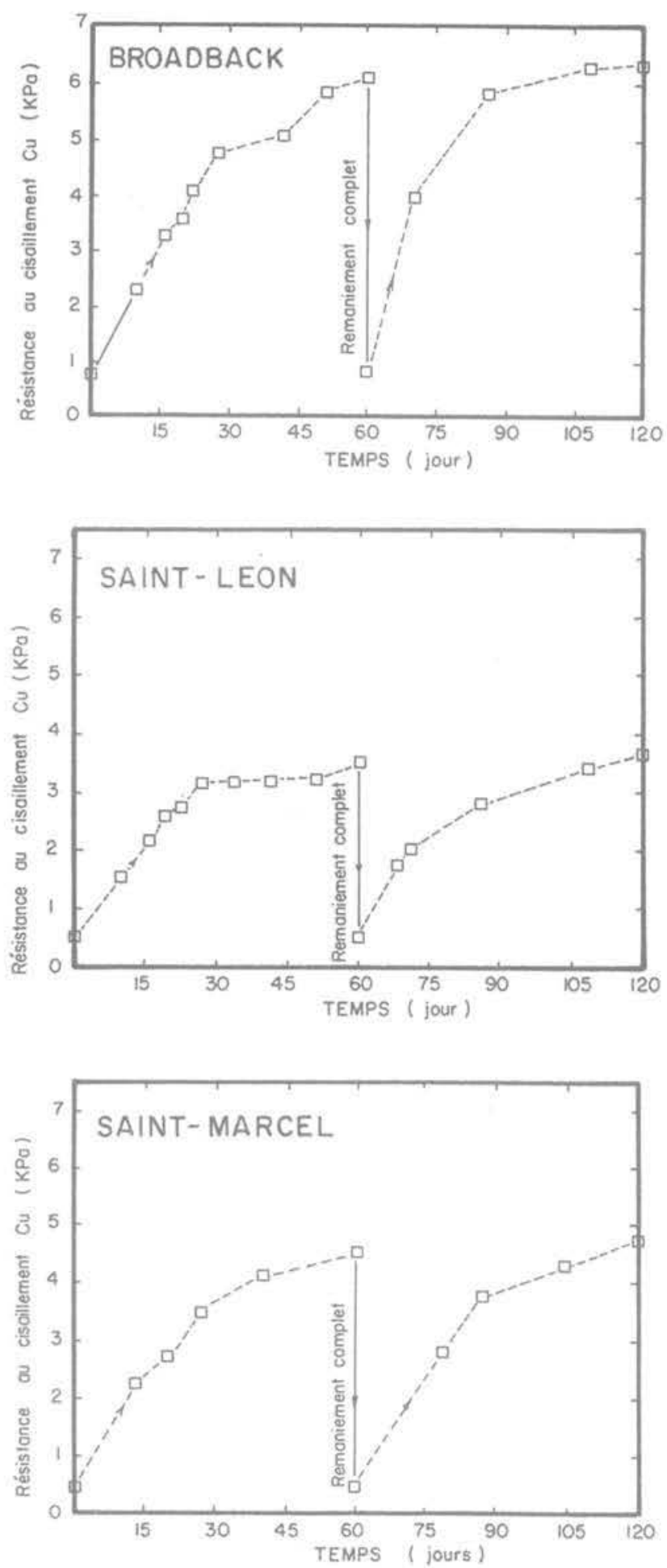

Fig. 4. - Gain de résistance au cisaillement du à la thixotropie en fonction du temps.

Fig. 4. - Increase in shear strength versus time, caused by thyxotropy.

De ces résultats, on retient que les valeurs des gains de résistance dus à la thixotropie pour ces trois argiles sont relativement importantes particulièrement pour l'argile de Broadback. 


\subsection{Comparaison entre le $c_{u}$ mesuré au cône suédois et le $c_{u}$ évalué en cisaillement simple ầ volume constant}

La figure 5 compare les valeurs de $c_{u}$ évaluées en cisaillement simple à volume constant lessai non drainé par analogie à l'essai triaxial) aux valeurs de c obtenues au cône suédois pour cinq échantillons consolidés à des pressions égales à 60 et $92,5 \mathrm{kPa}, 7$ et $60 \mathrm{kPa}$ et $15,5 \mathrm{kPa}$ respectivement pour l'argile de Broadback, de Saint-Léon et de Saint-Marcel. Il est important de préciser que c'était les mêmes échantillons remaniés et consolidés, une première fois, dans la cellule (fig. 2) qui furent soumis à l'essai de cisaillement simple à volume constant, après une période de reconsolidation aux mêmes pressions que celles qu'ils avaient subies dans la cellule; ces essais de cisaillement effectués à vitesse de déplacement horizontal égale à $0,59 \times 10^{-2} \mathrm{~mm} / \mathrm{mn}$ ont eu lieu juste après l'évaluation de $c_{u}$ au cône suédois. Les résultats obtenus (fig. 5) montrent que ces deux types d'évaluation de $c_{u}$ donnent des valeurs assez proches. Un résultat analogue a été aussi obtenu pour les argiles intactes de Broadback (LEFEBVRE, 1981) et de Saint-Léon (MORISETTE, 1983). Dans ces cas, la pression de consolidation appliquée sur les échantillons, lors de l'étape de consolidation, dans l'appareil de cisaillement est égale à la contrainte verticale effective in situ $\left(\sigma_{\mathrm{vo}}^{\prime}\right)$.

Les essais de cisaillement à volume constant ont également impliqué trois autres échantillons, dont un pour chaque argile étudiée, préalablement remaniés et consolidés dans la cellule. Dans ce cas, la pression de consolidation, lors de l'étape de consolidation, fut volontairement augmentée à environ $150 \mathrm{kPa}$, car le montage

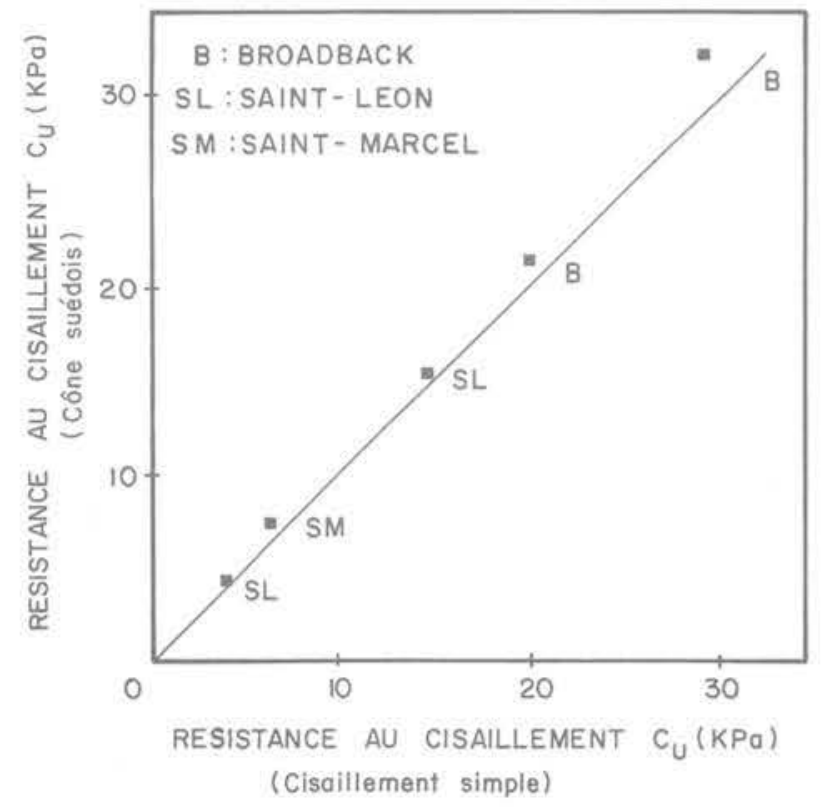

Fig. 5. - Comparaison de la résistance au cisaillement évaluée au cône suédois à celle évaluée au cisaillement simple à volume constant.

Fig. 5. - Comparison of the shear strength values evaluated by the swedish cone and the simple shear test at constant volume. de la cellule de consolidation utilisé ne permet pas l'usage de pressions supérieures à $100 \mathrm{kPa}$ sans de grandes déformations. Les valeurs de $c_{u}$ obtenues sont égales à $45,39,5$ et $36 \mathrm{kPa}$ respectivement pour Broadback, Saint-Léon et Saint-Marcel ; les valeurs du rapport $c_{v} / \sigma^{\prime}$ correspondant sont respectivement égales à 0,30 , 0,25 et 0,24 .

\subsection{Gain de résistance avec la consolidation}

Les essais de pénétration au cône suédois effectués sur les échantillons consolidés des argiles étudiées ont indiqué que la résistance au cisaillement $c_{4}$ évolue en fonction de la pression de consolidation $\sigma^{\prime}$, de la façon suivante: la résistance au cisaillement croît d'abord assez fortement avec laugmentation de la contrainte de consolidation, puis elle demeure stable dans une certaine plage de contrainte et, enfin, elle s'accroît pratiquement de façon proportionnelle à $\sigma^{\prime}$.

Ce comportement similaire au passage d'un sol structuré à normalement consolidé, a été particulièrement bien marqué pour les argiles de Broadback (fig. 6a) et Saint-Léon (fig. 7a) et à un moindre degré pour l'argile de Saint-Marcel (fig. 8a).

Les variations du rapport $c_{u} / \sigma^{\prime}$, et de la teneur en eau $w_{1}-w_{i}$ ( $w_{1}=$ teneur en eau initiale de l'échantillon lors de la mise en place dans la cellule de consolidation, $w_{\mathrm{f}}=$ teneur en eau de l'échantillon après consolidation) en fonction de $\sigma^{\prime}$ sont illustrées respectivement dans les figures $6 \mathrm{~b}$ à $8 b$ et $6 c$ à $8 c$ pour les trois argiles étudiées.

L'argile de Broadback présente une forte augmentation de $c_{y}$ pour les valeurs de $\sigma_{y}^{\prime}$ inférieures à $35 \mathrm{kPa}$ (fig. 6a). Ce comportement peut être associé aux pro. cessus suivants :

1. Un gain appréciable de la résistance au cisaillement par l'effet de thixotropie. Les essais de thixotropie, discutés précédemment, ont été réalisés à teneur en eau constante, ce qui n'est pas le cas d'un échantillon en cours de consolidation où la diminution de la teneur en eau risque d'atténuer le gain de résistance dû à la thixotropie (SKEMPTON et NORTHEY, 1952). Toutefois, on peut considérer cet effet négligeable et en tenant compte du paramètre temps (le temps de conservation de l'échantillon pour l'essai de thixotropie est égal à la durée de l'essai de consolidation), la valeur du gain thixotropique de résistance correspondant à $\sigma_{y}^{\prime}=7 \mathrm{kPa}, 15,5 \mathrm{kPa}$ et $32,5 \mathrm{kPa}$ est respectivement égal à $2,5 \mathrm{kPa}, 3,0 \mathrm{kPa}$ et $3,25 \mathrm{kPa}$ ce qui représente, par rapport aux valeurs de $c_{u}$ respectives, les pourcentages suivants : $33,5,22$ et 15 . Ceci démontre que l'effet de thixotropie est fort important du moins pour cette plage de contraintes de consolidation.

2. Une forte diminution de la teneur en eau, $w_{p}$ pour la plage de pression $7 \mathrm{kPa}$ à $32,5 \mathrm{kPa}$, (fig. $6 \mathrm{c}$ ); au-delà de $\sigma_{v}^{\prime}=32,5 \mathrm{kPa}$, la teneur en eau $w_{f}$ diminue à un taux nettement inférieur.

Ainsi, les effets de la thixotropie et de la compression importante pour $\sigma_{v}^{\prime}$ inférieure à $32,5 \mathrm{kPa}$ semblent res- 
ARGILE BROADBACK
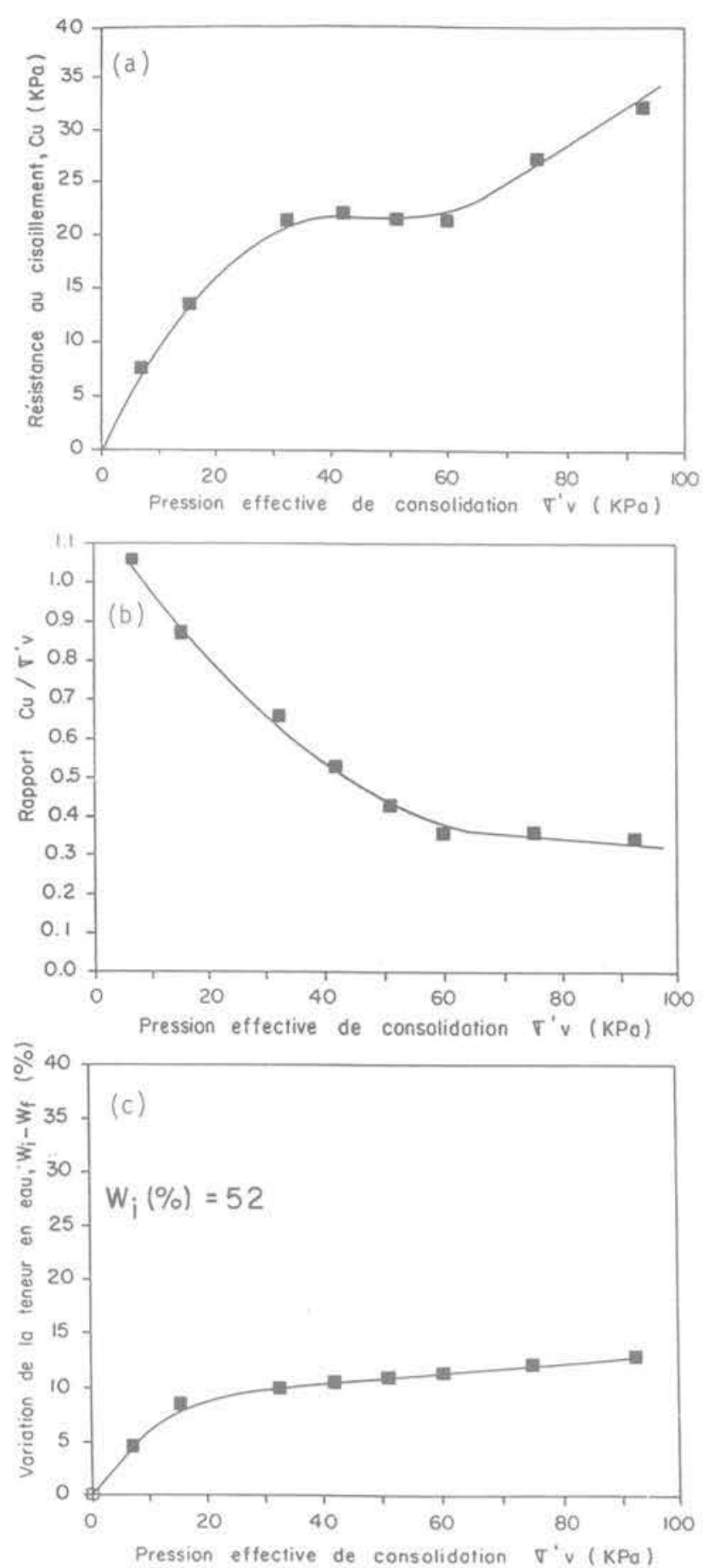

Fig. 6. - Résultats obtenus sur l'argile de Broadback.

(a) Variation de la résistance au cisaillement en fonction de la pression de consolidation.

(b) Variation du rapport $c_{u} / \sigma_{v}^{\prime}$ en fonction de la pression de consolidation.

(c) Variation de la teneur en eau $w_{f}-w_{f}$ en fonction de la pression de consolidation.

Fig. 6. - Results on Broadback clay.

(a) Shear strength change versus consolidation pressure.

(b) Change of the ratio $c_{u} / \sigma_{v}^{\prime}$ versus consolidation pressure.

(c) Change of the water content $w_{t}-w_{f}$ versus consolidation pressure.
ARGILE SAINT-LEON
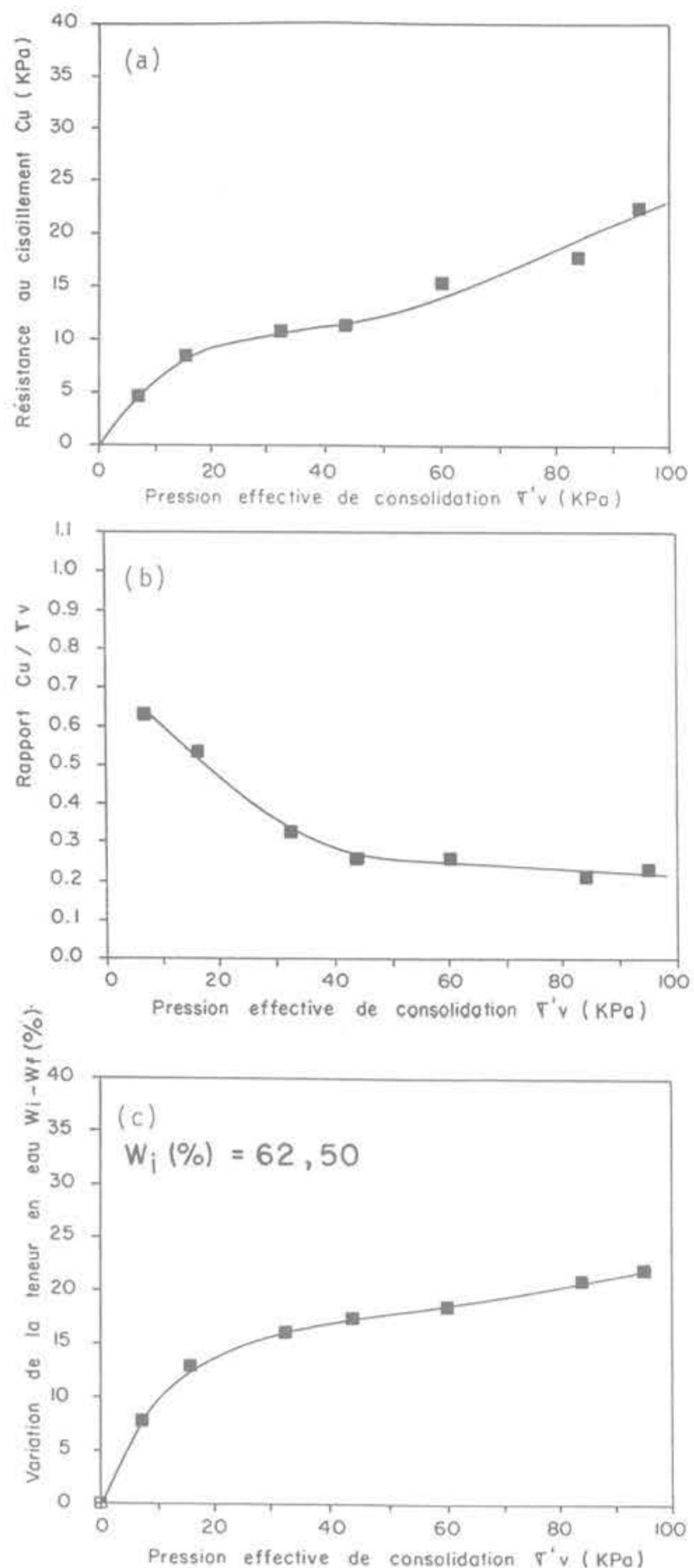

Fig. 7. - Résultats obtenus sur l'argile de Saint-Léon.

(a) Variation de la résistance au cisaillement en fonction de la pression de consolidation.

(b) Variation du rapport $c_{u} / \sigma_{v}^{\prime}$ en fonction de la pression de consolidation.

(c) Variation de la teneur en eau $w_{i}-w_{f}$ en fonction de la pression de consolidation.

Fig. 7. - Results on Saint-Léon clay.

(a) Shear strength change versus consolidation pressure.

(b) Change of the ratio $c_{u} / \sigma_{v}$ versus consolidation pressure.

(c) Change of the water content $w_{i}-w_{f}$ versus consolidation pressure. 
ARGILE SAINT-MARCEL
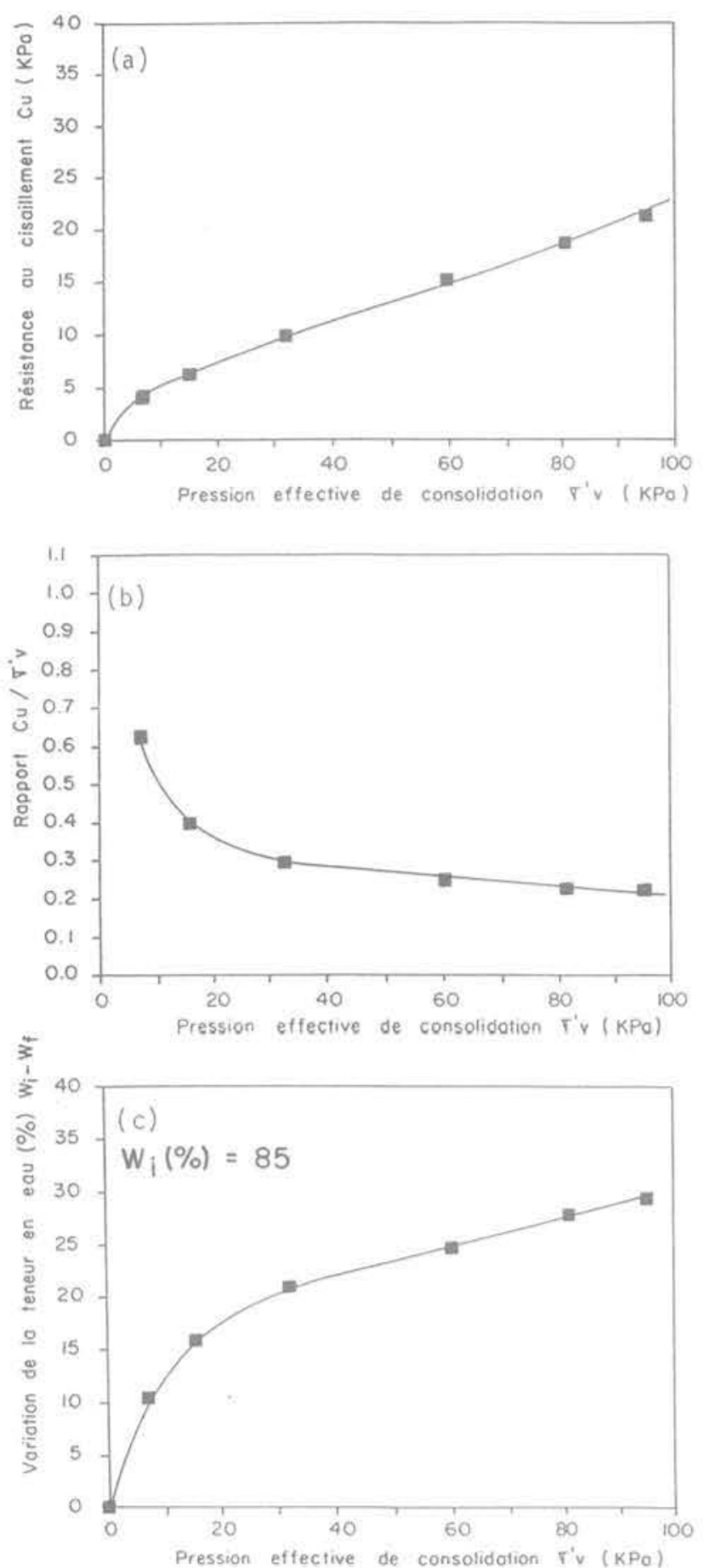

Fig. 8. - Résultats obtenus sur I'argile de Saint-Marcel.

(a) Variation de la résistance au cisaillement en fonction de la pression de consolidation.

(b) Variation du rapport $c_{u} / \sigma_{v}^{\prime}$ en fonction de la pression de consolidation.

(c) Variation de la teneur en eau $w_{i}-w_{f}$ en fonction de la pression de consolidation.

Fig. 8. - Results on Saint-Marcel clay.

(a) Shear strength change versus consolidation pressure.

(b) Change of the ratio $c_{u} / \sigma_{v}^{\prime}$ versus consolidation pressure.

(c) Change of the water content $w_{1}-w_{t}$ versus consolidation pressure. ponsables de la forte augmentation de $c_{u}$ de l'argile Broadback. Ces deux facteurs paraissent également responsables de la forte augmentation de c pour $\sigma^{\prime}$, inférieures à $15,5 \mathrm{kPa}$ pour l'argile de Saint-Léon (fig. 7a) et pour l'argile de Saint-Marcel (fig. 8a).

De plus, pour l'argile de Broadback, c demeure plus ou moins stable dans la plage de pression comprise entre $32,5 \mathrm{kPa}$ et $60 \mathrm{kPa}$ (fig. $6 \mathrm{a}$ ). En effet, $\mathrm{c}$ oscille entre $21,5 \mathrm{kPa}$ et $22 \mathrm{kPa}$ alors que les échantillons ont été consolidés sous des valeurs de $\sigma^{\prime}$, égales à 32,5 , 42,5 et $60 \mathrm{kPa}$. Les causes d'un tel comportement ne sont pas identifiables clairement; ceci pourrait être dû à une structuration de l'argile qui aurait pris place à $\sigma$ ' inférieure à $30 \mathrm{kPa}$. Cet effet de structure (liens ou enchevêtrements de particules du sol) disparaitrait vers $60 \mathrm{kPa}$. Au-delà de cette valeur, $\mathrm{c}$ augmente de nouveau et ce de façon proportionnelle à $\sigma_{\text {, comme pour }}$ une argile normalement consolidée, avec un rapport $c_{v} / \sigma^{\prime}$ égal à environ 0,35 (fig, $6 \mathrm{~b}$ ). Ce seuil de $60 \mathrm{kPa}$ peut être considéré comme la pression de destructu. ration de cette argile remaniée.

\section{MÉCANISME DE COMPORTEMENT D'UNE ARGILE REMANIÉE ET CONSOLIDÉE}

Les résultats des essais de consolidation réalisés sur ces trois argiles remaniées permettent d'identifier un mécanisme de comporternent, $c_{\mathrm{u}}$ versus $\sigma$, illustré dans la figure 9; un résumé des caractéristiques mécaniques de ces trois argiles, associées à ce mécanisme de comportement, est présenté dans le tableau III. Aussi l'évolution

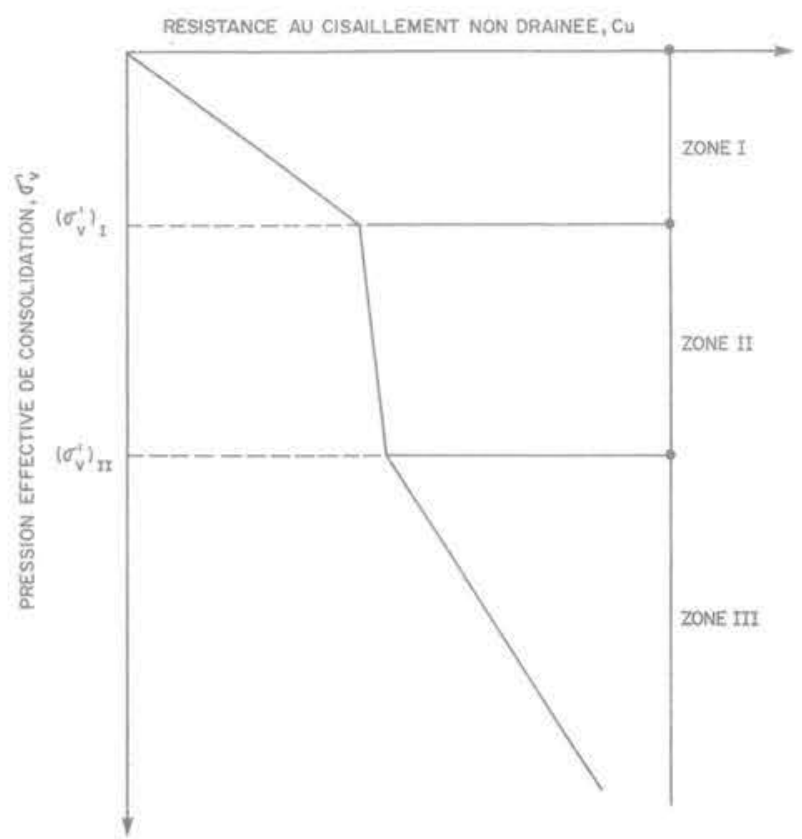

Fig. 9. - Mécanisme de comportement d'une argile remaniée et consolidée.

Fig. 9. - Behavior mechanisms for a remoulded consolidated clay. 
Tableau III. - Résumé des caractéristiques relatives aux trois zones (zones I, II et III) du mécanisme de comportement illustré à la figure 9.

Table III. - Summary of the 3 zones (zones I. II and III) characteristics on behavior mechanisms shown in figure 9 .

\begin{tabular}{|c|c|c|c|c|}
\hline & & Broadback & Saint-Léon & Saint-Marcel \\
\hline $\begin{array}{c}\text { Fin Zone I } \\
\text { Début Zone II }\end{array}$ & $\begin{array}{l}\left(\sigma_{v}^{\prime}\right),(\mathrm{kPa}) \\
\left(\mathrm{c}_{u}\right),(\mathrm{kPa}) \\
\left(\mathrm{cu}_{1}\right),(\mathrm{kPa}) \\
\left(\mathrm{c}_{u} / \sigma_{v}^{\prime}\right) \\
\left(\mathrm{w}_{1}-\mathrm{w}_{f}\right),(\%)\end{array}$ & $\begin{array}{l}30 \\
21 \\
3,25 \\
0,70 \\
9,9\end{array}$ & $\begin{array}{c}15 \\
8,5 \\
1,40 \\
0,56 \\
12,9\end{array}$ & $\begin{array}{l}15 \\
6,2 \\
1,60 \\
0,47 \\
16,0\end{array}$ \\
\hline $\begin{array}{l}\text { Fin Zone II } \\
\text { Début Zone III }\end{array}$ & $\begin{array}{l}\left(\sigma_{v}^{\prime}\right)_{11}(\mathrm{kPa}) \\
\left(\mathrm{c}_{u}\right)_{\| 1}(\mathrm{kPa}) \\
\left(\mathrm{cu}_{u}\right)_{11}(\mathrm{kPa}) \\
\left(\mathrm{c}_{u} / \sigma_{\mathrm{v}}^{\prime}\right)_{11} \\
\left(w_{i}-w_{1}\right)_{\|}(\%)\end{array}$ & $\begin{array}{l}60 \\
21,5 \\
4,0 \\
0,35 \\
11,4\end{array}$ & $\begin{array}{l}45 \\
10,35 \\
2,2 \\
0,23 \\
17,5\end{array}$ & $\begin{array}{l}45 \\
9,9 \\
2,2 \\
0,22 \\
22,9\end{array}$ \\
\hline
\end{tabular}

$w_{i}=52,62,5$ et $85 \%$ respectivement pour Broadback, Saint-Léon et Saint-Marcel.

de $c_{u}$ en fonction de $\sigma_{v}^{\prime}$ se décompose en trois zones distinctes:

1. Zone I: $c_{u}$ croit fortement en fonction de l'augmentation de $\sigma_{v}^{\prime}$ ce qui correspond à des rapports $c_{v} / \sigma_{v}^{\prime}$ élevés (tableau III). Dans cette zone on a observé:

- une forte diminution de la teneur en eau et donc de l'indice des vides, ce qui se traduirait par une aug. mentation de résistance au cisaillement due à la réduc. tion des vides comme dans les matériaux pulvérulents grossiers ;

- un gain appréciable de résistance dû à l'effet de thixotropie. Le tableau III montre l'importance de ces deux aspects par les variations de la teneur en eau $\left(w_{i}-w_{p}\right)$ et du gain de résistance dû à la thixotropie $\left(c_{u t}\right)$.

Les valeurs du rapport $c_{w} / \sigma_{v}^{\prime}$ à la fin de la zone 1 , $\left(\left(c_{1} / \sigma^{\prime}\right) I\right)$ sont de $0,7,0,56$ et 0,41 respectivement pour Broadback, Saint-Léon et Saint-Marcel. A une faible pression de consolidation $\sigma^{\prime}$ égale à $7 \mathrm{kPa}$, le rapport. $c_{u} / \sigma_{v}^{\prime}$ atteint des valeurs aussi élevées que 1,05, 0,65 et 0,63 respectivement pour les argiles de Broadback (fig. 6b), de Saint-Léon (fig. 7b), Saint-Marcel (fig. 8b).

Or, ce rapport $c_{u} / \sigma_{v}^{\prime}$ se situe généralement pour les argiles marines normalement consolidées entre 0,17 et 0,3 (BJERRUM and SIMONS, 1960); ces résultats permettent donc d'affirmer que l'augmentation de la résistance au cisaillement observée dans cette zone I, pour les trois argiles étudiées, n'est pas due uniquement à la pression de consolidation appliquée $\sigma^{\prime}$. Une partie de ce gain de résistance doit être attribuée à la résistance du squelette argileux due au développement de nouveaux liens ou enchevêtrements au niveau des particules du sol. Dans les dépôts de l'Est du Canada, on attribue souvent cette résistance au cisaillement due à un effet autre que la consolidation à une structuration ou une cimentation qui se serait développée avec les siècles durant ou après la formation des dépôts (LOCAT, 1982). Ainsi les valeurs du rapport $c_{y} / \sigma_{y}^{\prime}$ élevées de cette zone 1 montrent que les trois argiles étudiées pos. sèdent une "résistance de réserve » en cisaillement (BROWN and RASHID, 1975) et qu'elles devraient être décrites comme des argiles « surconsolidées ». Cette surconsolidation provient d'une structuration des argiles due à une grande compression initiale, comme men. tionné auparavant. Cette structuration est développée tout au long de la zone I qui semble prendre fin à $\sigma^{\prime}$ égal à $30 \mathrm{kPa}$ pour l'argile de Broadback et à $\sigma^{\prime}$ égal à $15 \mathrm{kPa}$ pour les argiles de Saint-Léon et de SaintMarcel (tableau III ; fig. 6a à 8a). Aussi cette surconsolidation est confirmée par les résultats d'essais de cisaillement simple à volume constant réalisés sur des échantillons d'argile de Saint-Léon (fig, 10). En effet, cette figure montre que le cheminement de contraintes $\tau_{h}-\sigma_{v}^{\prime}$ correspondant à l'échantillon consolidé à $\sigma_{v}^{*}=7 \mathrm{kPa}\left(\mathrm{c}_{\mathrm{u}} / \sigma_{\mathrm{v}}^{\prime}=0,63\right)$ montre un comportement d'une argile surconsolidée, alors que ceux correspondant aux deux autres essais réalisés à $\sigma^{\prime}=60$ et $152 \mathrm{kPa}\left(c_{4} / \sigma^{\prime}=0,26\right.$ et 0,25 , respectivement) montrent un comportement d'une argile normalement consolidée.

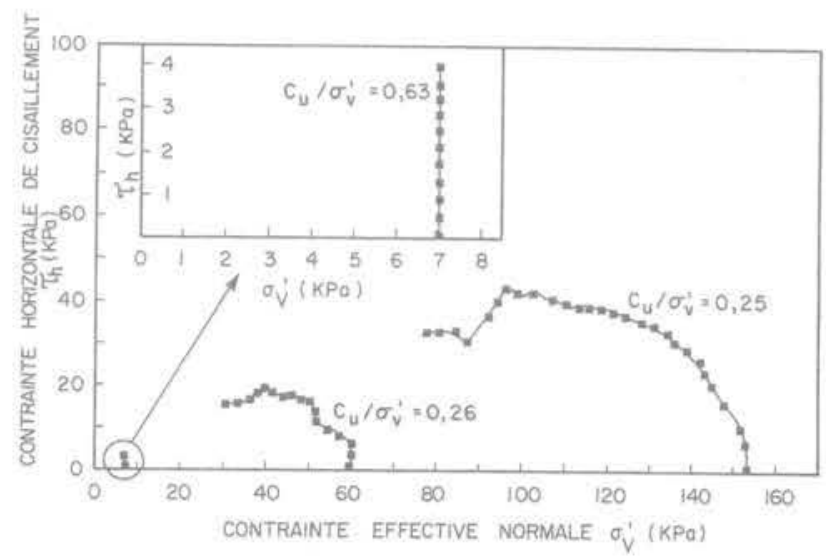

Fig. 10. - Cheminements-vecteurs résultant d'essais de cisaillement simple à volume constant effectués. sur des échantillons d'argile de Saint-Léon.

Fig. 10 - Stress paths from simple shear tests at constant volume on Saint-Léon clay 
2. Zone II : $\mathrm{c}_{\mathrm{u}}$ croît très peu en fonction de laugmentation de $\sigma_{\mathrm{v}}^{\prime}$. Ceci est dû à une structuration qui s'est développée précédemment (zone I). Cette structuration disparait par la suite graduellement tout au long de la zone II, car la résistance maximale développée par le squelette argileux est atteinte. La pression de consolidation qui correspond à la fin de cette zone, $\left(\sigma^{\prime}\right)_{1}$, est de $60 \mathrm{kPa}$ pour l'argile de la mer de Tyrrell et de $45 \mathrm{kPa}$ pour celle de la mer de Champlain; une fois ces valeurs atteintes (début de la zone III), l'accroisse. ment de $c_{u}$ en fonction de $\sigma_{y}^{\prime}$ est nettement supérieur, l'effet de structuration du squelette semble effacé.

Dans cette zone II, les courbes de compressibilité et de variations de la perméabilité présentées ailleurs (BEN. MOKRANE, 1983) n'ont montré aucune évolution particulière; le changement de volume d'eau a été continu, comme on le constate sur les figures $6 \mathrm{c}$ à $8 \mathrm{c}$. Aussi les caractéristiques de cette deuxième zone, résumées dans le tableau IV, montrent que:

- l'étendue de cette phase, représentée par la plage de contrainte $\left(\sigma_{v}^{\prime}\right)_{11}-\left(\sigma_{v}^{\prime}\right)_{1}$ est sensiblement la même pour les trois argiles étudiées ici; elle vaut environ $30 \mathrm{kPa}$. Ces argiles sont toutes les trois d'origine glaciaire et elles se sont déposées en milieu marin ou saumâtre. Leurs compositions minéralogiques sont analogues; elles résultent de l'érosion du bouclier laurentien lors de la dernière glaciation (Wisconsin). Ces éléments communs permettent d'expliquer les analogies observées dans la définition de la zone II ;

- l'effet de la structuration sur les variations de c dans la zone II fut plus important pour l'argile de la mer de Tyrrell (argile de Broadback). En effet, malgré une variation de contrainte de $\sigma_{v}^{\prime}=30 \mathrm{kPa}$, la résistance au cisaillement n'a pratiquement pas augmentée $\left(\Delta c_{u}<0,5 \mathrm{kPa}\right)$. Les deux argiles de la mer de Champlain ont montré une légère augmentation de la résistance au cisaillement soit $\Delta c_{u}$ égal à $1,85 \mathrm{kPa}$ (Saint-Léon) et $3,70 \mathrm{kPa}$ (Saint-Marcel). Ces résultats indiquent qu'une valeur $\Delta \mathrm{c}_{\mathrm{u}}$ faible correspond à une valeur de $\left(c_{n} / \sigma^{\prime}\right)$, élevée (tableau III). Ainsi, lorsque la valeur de $\left(c_{\mathrm{u}} / \sigma_{\mathrm{v}}^{\prime}\right)_{1}$ est très forte, le cas de l'argile de la mer de Tyrrell $(0,7)$, on observe pratiquement un plateau dans la zone II. Le tableau I indique des pourcentages en minéraux argileux (phyllo-silicates) de $75 \%$ (Broadback), 25\% (Saint-Léon) et 35\% (Saint-Marcel) ; parallèlement, on note que la teneur en matières amorphes augmente avec ce pourcentage de phyllosilicates (BALLIVY et al., 1971). Ces variations pourraient expliquer les différentes valeurs de $c_{\mathrm{u}}$ dans cette zone II qui correspondrait à la résistance du squelette due à une consolidation accrue et aussi au développement de résistance par cimentation due aux composés amorphes ou aux liaisons de Van der Waals entre les phyllosilicates. Par ailleurs, lors de la formation des dépôts d'argile, la thixotropie pourrait se développer dans la zone II et être d'autant plus importante que la compression (indiquée ici par le changement de la teneur en eau $w_{1}-w_{f}$ ) est faible dans cette zone, même si la déposition continue (plage zone II). Cette résistance additionnelle augmenterait le blocage de la structure. Avec le temps, la résistance de plateau dans la zone II pourrait donc être augmentée sur le terrain d'un gain thixotropique au moins égal à ce qui a été mesuré en laboratoire (fig. 4) soit $6,1,3,5$ et $4,5 \mathrm{kPa}$ respectivement pour Broadback, Saint-Léon et Saint-Marcel. Par gain thixotropique, on peut entendre toute augmentation de résistance avec le temps dont la possibilité de création de différents types de liens (matériaux amorphes, etc.).

3. Zone III, dans laquelle $\mathrm{c}_{u}$ crôt de façon proportionnelle à $\sigma^{\prime}$. La valeur de ce rapport c/ $\sigma^{\prime}$ est de 0,35 (Broadback), 0,23 (Saint-Léon) et 0,22 (Saint-Marcel). Le début de cette phase correspond à la disparition de l'effet de la structuration; la valeur correspondante de $\sigma^{\prime}$, est égale à environ $60 \mathrm{kPa}$ pour l'argile de la mer de Tyrrell et $45 \mathrm{kPa}$ pour les deux sites de la mer de Champlain. Dans cette troisième zone, les valeurs de $\sigma^{\prime}$ sont suffisantes pour permettre une croissance normale de la résistance au cisaillement $c_{u}$. Toute augmentation de $\sigma^{\prime}$ provoque une croissance de $c_{4}$ avec un rapport $c_{u} / \sigma_{\mathrm{v}}^{\gamma}$ de valeur analogue à celle correspondant généralement pour les argiles normalement consolidées.

\section{APPLICATION À L'ÉTUDE DES CONDITIONS IN SITU}

Les dépôts d'argile en cours de formation dans les fonds marins actuels jouent un rôle très important pour la conception des aménagements off-shore. Par exemple la mise en place de remblais ou d'équipements submergés implique la connaissance de la résistance au cisaillement des premiers mètres du fond marin. Si l'on utilise un rapport usuel de $c_{4} / \sigma^{\prime}$, de l'ordre de 0,22 par exemple, la résistance au cisaillement $c_{u}$ à un ou deux mètres de profondeur n'est alors que de l'ordre de quelques $\mathrm{kPa} ; \mathrm{c}_{4}$ est de l'ordre de $7 \mathrm{kPa}$ à deux mètres de profondeur pour un poids spécifique du sol

Tableau IV. - Résumé des caractéristiques relatives à la zone II des trois argiles marines étudiées. Table IV. - Summary of zone II characteristics for the three clays under study.

\begin{tabular}{|l|c|c|c|}
\hline \multirow{2}{*}{} & \multicolumn{2}{|c|}{ Mer de Tyrrel } & Mer de Champlain \\
\cline { 2 - 4 } & Broadback & Saint-Léon & Saint-Marcel \\
\hline$\left(\sigma_{v}^{\prime}\right)_{\|}-\left(\sigma_{v}^{\prime}\right),(\mathrm{kPa})$ & 30 & 30 & 30 \\
\hline$\left(c_{u}\right)_{\|}-\left(c_{u}\right),(\mathrm{kPa})$ & 0,5 & 1,85 & 3,70 \\
\hline$\left(w_{i}-w_{t}\right) \|-\left(w_{i}-w_{i}\right),(\%)$ & 1,5 & 4,6 & 6,9 \\
\hline
\end{tabular}


égal à $16 \mathrm{kN} / \mathrm{m}^{3}$. Dans ce cas-ci, la connaissance exacte du rapport $c_{v} / \sigma_{\text {, }}$ pour des très faibles contraintes verticales revêt donc une grande importance. Aussi, les essais de consolidation cedométrique appliqués à ces trois argiles marines remaniées montrent que le rapport $c_{1} / \sigma$ ' pour une très faible contrainte est au moins deux fois plus élevé que la valeur déterminée dans une plage de pression plus élevée, Donc la résistance au cisaillement $c_{u}$, pour de faibles contraintes, est au moins deux fois plus élevée que celle qui aurait été déterminée à partir d'un rapport $\mathrm{c}_{\mathrm{u}} / \sigma_{\mathrm{v}}^{\prime}$ définie à une plage de pression élevée.

Les résultats présentés ici ont par ailleurs montré qu'il faudrait s'attendre à une augmentation très faible de la résistance avec la profondeur dès que la profondeur correspondant à la fin de la zone I est atteinte (environ $30 \mathrm{kPa}$ pour l'argile de la mer de Tyrrell et $15 \mathrm{kPa}$ pour les deux argiles de la mer de Champlain). L'étendue de cette zone II, où $\mathrm{c}_{u}$ n'augmente que très faiblement correspond à une variation de contraintes $\sigma^{\prime}$ d'environ $30 \mathrm{kPa}$ et ce, pour les trois argiles étudiées.

JEFFERIES et al. (1985) ont compilé des données sur la résistance $c$ obtenue à partir de plusieurs essais en laboratoire et in situ dans un dépôt d'argile en voie de formation dans les fonds de la mer de Beaufort. Le profil de résistance qu'ils ont obtenu est aussi caractérisé par les trois zones identifiées précédemment, soit une zone I dans laquelle $c$ augmente fortement avec la profondeur, une zone II dans laquelle $c_{u}$ augmente très faiblement ou pratiquement pas avec la profondeur et enfin une zone III dans laquelle $c_{u}$ croît en fonction de la profondeur. Un résultat analogue a été aussi obtenu par MORIN et DAWE (1986) pour un dépôt d'argile marine dans les fonds de la mer du Labrador.

Les variations de $c_{\mathrm{u}}$ en fonction de $\sigma_{\mathrm{y}}$, obtenues en laboratoire peuvent aussi être comparées aux profils de la résistance des dépôts définis en place aux sites de Broadback et de Saint-Marcel; le dépôt d'argile de Saint-Léon n'est pas considéré, car il semble avoir connu une très forte surconsolidation géologique (MORISSETTE, 1983). La résistance $c_{u}$ des dépôts en place a été évaluée à l'aide d'un scissomètre de type Nilcon (LEFEBVRE, 1981).

\subsection{Dépôt d'argile de la mer de Tyrrell (Broadback)}

Les deux profils de $c_{u}$ (laboratoire et dépôt) sont illustrés dans la figure 11. Le profil de $\mathrm{c}_{u}$ du dépôt naturel est également constitué de trois parties:

- une première partie où $c_{u}$ diminue en fonction de la profondeur, caractérisée par la couche altérée de surface (croûte) allant jusqu'à $5 \mathrm{~m}$ de profondeur. A ce niveau $(5 \mathrm{~m})$, la pression effective naturelle $\sigma_{\text {vo }}^{\prime}$ et la pression de pré-consolidation $\sigma_{\mathrm{p}}^{\prime}$ sont respectivement égales à $40 \mathrm{kPa}$ et $150 \mathrm{kPa}$;

- une deuxième partie où $c_{u}$ demeure constant en fonction de la profondeur $(5 \mathrm{~m}-11 \mathrm{~m})$. Dans cette zone $(5 \mathrm{~m}-11 \mathrm{~m})$ la surconsolidation est à peu près constante à $90 \mathrm{kPa}$. A une profondeur de $11 \mathrm{~m}$, on a $\sigma_{\mathrm{vo}}^{\prime}=90 \mathrm{kPa}$ et $\sigma_{\mathrm{p}}^{\prime}=180 \mathrm{kPa}$;

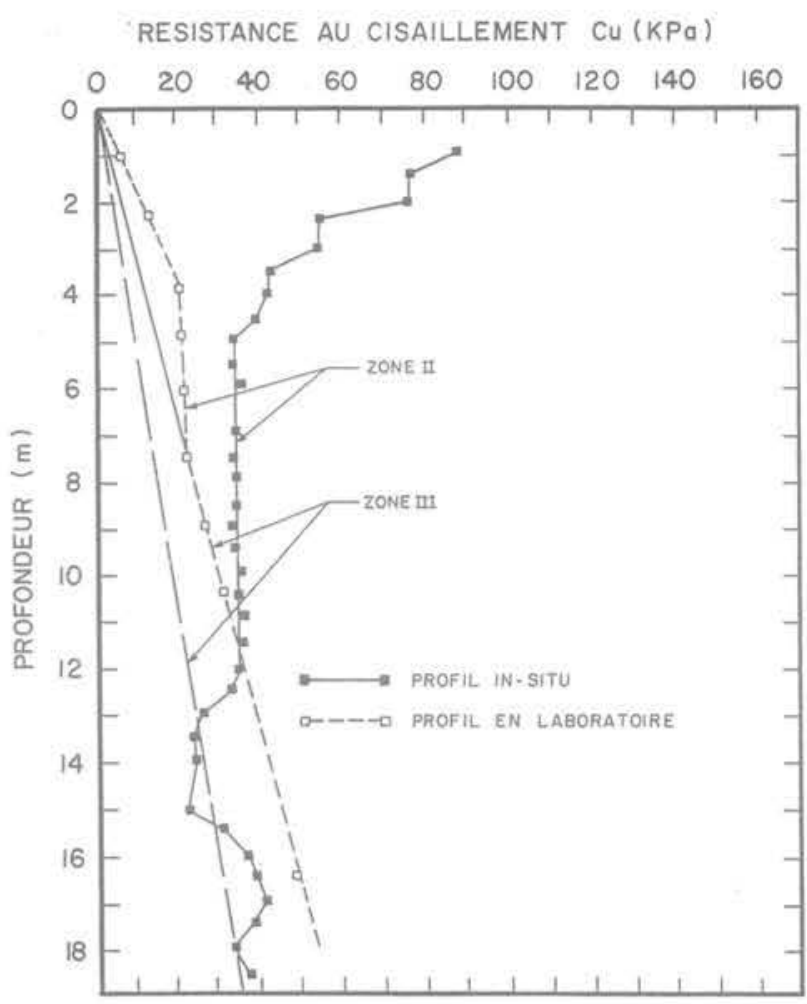

Fig. 11. - Comparaison du profil de $c_{u}$ obtenu en laboratoire à celui évalué sur le dépôt - Argile de Broadback.

Fig. 11. $-c_{y}$ profile obtained in laboratory compared to the $c_{v}$ profile evaluated in situ - Broadback clay.

- une troisième partie (à partir de $16 \mathrm{~m}$ ) où $\mathrm{c}_{\mathrm{u}}$ augmente en fonction de la profondeur et qui est caractérisée par un rapport $c_{\mathrm{u}} / \sigma_{\mathrm{vo}}^{\prime}$ à peu près constant et égal à 0,19 . Dans cette zone, l'argile a un comportement de type normalement consolidé. La couche de sol comprise entre $11 \mathrm{~m}$ et $16 \mathrm{~m}$ est une zone de transition dans les conditions de sédimentation (présence de silt, sable et gravier); le dépôt d'argile sous-jacent étant un dépôt d'origine lacustre (Lac Barlow-Ojibway).

Ainsi, le profil de $c_{u}$ (laboratoire) et le profil de $c_{u}$ (dépôt) montrent un comportement plus ou moins similaire. L'écart entre les valeurs de $c_{\text {u }}$ de la zonel (laboratoire et dépôt) est relié essentiellement aux effets combinés de l'altération de l'oxydation. La différence entre $\left(\sigma_{v}^{\prime}\right)=30 \mathrm{kPa}$ et $\sigma_{\mathrm{p}}^{\prime}=150 \mathrm{kPa}$ est également associée à ces mêmes efféts (altération et oxydation). La zone II (laboratoire) est similaire à la deuxième partie (dépôt). La surconsolidation $\left(\sigma_{p}^{\prime}-\sigma_{v_{0}}^{\prime}=90 \mathrm{kPa}\right)$ ré. gnant dans cette partie $\left(5 \mathrm{~m}-{ }^{\mathrm{p}} 11 \mathrm{~m}\right)$ peut être due à une cimentation d'origine chimique ou électrostatique, ou à des enchevêtrements plus importants des particules dans le dépôt ou encore à l'effet du temps de consolidation (BJERRUM, 1967). Le dépôt de Broadback date, en effet, d'environ 8000 ans et cet effet du temps ne peut être totalement recréé dans ces essais. La différence, $\Delta c_{u}=12 \mathrm{kPa}$, entre $c_{u}$ (Zone II) et $c_{u}$ (deuxième partie) peut être due aux mêmes phénomènes. Il en va de même pour l'écart entre $\left(\sigma_{\text {v }}^{\prime}\right)_{11}=60 \mathrm{kPa}$ et $\sigma^{\prime}=180 \mathrm{kPa}$. Finalement, les zones III sont similaires. L'écart entre les valeurs de $\mathrm{c}_{\mathrm{v}} /$ 
$\sigma_{y}^{\prime}=0,35$ (laboratoire) et 0,19 (dépôt), est en partie dû́ aussi à des variations dans les caractéristiques sé. dimentologiques du dépôt comparativement à celles de l'échantillon utilisé en laboratoire.

\subsection{Argile de la mer de Champlain (Saint-Marcel)}

Les deux profils de c (laboratoire et dépôt) sont représentés à la figure 12. Le profil de $c_{u}$ du dépôt est constitué de trois parties

- une partie caractérisée par la croûte altérée de surface dans laquelle $c$ diminue en fonction de la profondeur, jusqu'à $2 \mathrm{~m}$ de profondeur. A ce niveau, $\sigma^{\prime}$ et $\sigma_{\mathrm{p}}^{\prime}$ valent respectivement 27 et $48 \mathrm{kPa}$;

- une deuxième partie, située entre $2 \mathrm{~m}$ et $7 \mathrm{~m}$, dans laquelle $c_{u}$ croît légèrement avec la profondeur. La résistance au cisaillement passe de $13 \mathrm{kPa}$ à la base de la croûte $(2 \mathrm{~m})$ à environ $18 \mathrm{kPa}$ à $7 \mathrm{~m}$ de profondeur. On a donc seulement une augmentation de résistance de $5 \mathrm{kPa}$ pour une variation de profondeur égale à $5 \mathrm{~m}$. Les valeurs de $\sigma_{\text {vo }}^{\prime}$ et $\sigma_{p}^{\prime}$ au niveau $7 \mathrm{~m}$ valent respectivement $56 \mathrm{kPa}$ et $76 \mathrm{kPa}$. L'argile dans cette deuxième zone est légèrement surconsolidée, $\sigma^{\prime}-\sigma^{\prime}$ d'environ $20 \mathrm{kPa}$ et un rapport $\sigma^{\prime} / \sigma_{\mathrm{vo}}^{\prime}$ moyen de 1,6 . Cette deuxième partie du profil de résistance du dépôt semble donc avoir la même allure que la zone II (laboratoire) mais plus amplifiée sur le terrain probablement à cause de liens de structuration plus forts qui se sont développés depuis la déposition (12000 ans pour le dépôt de Saint-Marcel) ;

- une troisième partie dans laquelle c augmente en fonction de la profondeur $(7 \mathrm{~m}-$ fin de forage $(10 \mathrm{~m})$ ). Ainsi $c_{\mathrm{u}}$ passe de $18 \mathrm{kPa}$ à $7 \mathrm{~m}$ de profondeur à $25 \mathrm{kPa}$

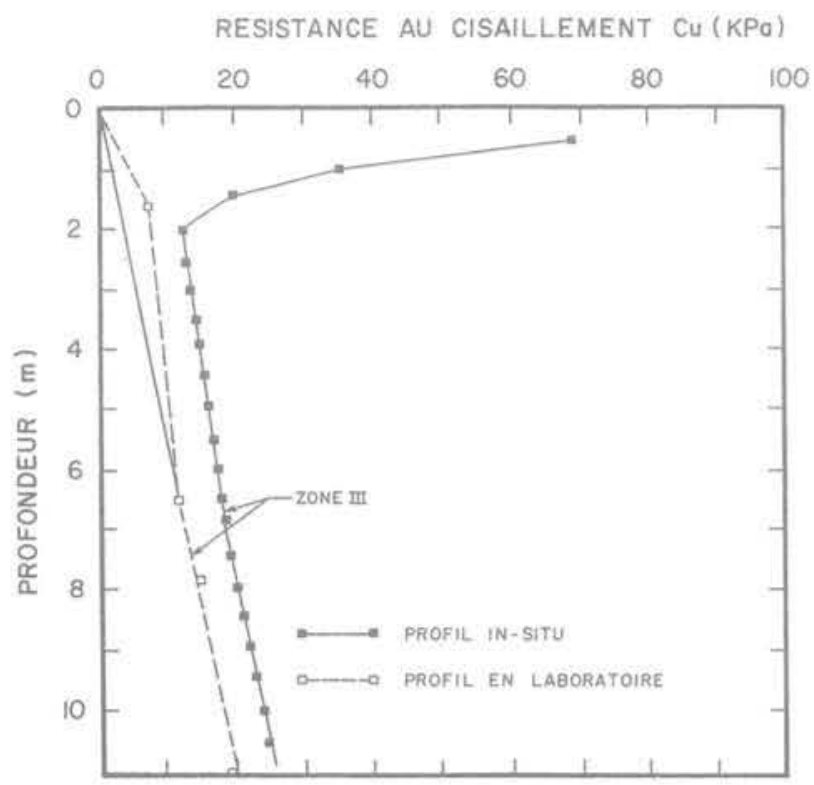

Fig. 12. - Comparaison du profil de $c_{u}$ obtenu en laboratoire à celui évalué sur le dépôt - Argile de Saint-Marcel.

Fig. 12. $-c_{u}$ profile obtained in laboratory compared to the $c_{i}$ profile evaluated in situ - Saint-Marcel clay. à $10 \mathrm{~m}$ de profondeur. Dans cette zone, le rapport $c_{v} / \sigma_{p}^{\prime}$ est peu constant, et égal à 0,27 et l'argile est légèrement surconsolidée, $\sigma_{p}^{\prime}-\sigma_{v o}^{\prime}$ d'environ $18 \mathrm{kPa}$ et un rapport $\sigma^{\prime} / \sigma_{v p}^{\prime}$ moyen de 1,3 . Si l'on considère que $c_{4} / \sigma_{p}^{\prime}$ demeure constant à 0,27 lorsque le dépôt devient normalement consolidé, on constate que cette valeur est proche de la valeur de $c_{\nu} / \sigma^{\prime}$ de la zone III (laboratoire) qui est de 0,22 .

\section{CONCLUSION}

Des échantillons de trois argiles marines du Québec (Canada), dont une provenant de la mer de Tyrrell (site de Broadback) et les deux autres de la mer de Champlain (sites de Saint-Léon et Saint-Marcel), ont été remaniés et ensuite soumis à des essais de reconsolidation cedométrique sous de faible contraintes variant de 7 à $150 \mathrm{kPa}$. Ce niveau de contrainte de consolidation tra. duit ainsi des conditions éventuelles dans des dépôts récents encore immergés. Les échantillons reconsolidés ont été soumis à des essais de pénétration au cône suédois pour évaluer la résistance au cisaillement non drainée $c_{u}$, ce qui a permis l'étude de la relation $c_{4}-\sigma^{\prime}$. Aussi, des essais de cisaillement simple à volume constant ont été effectués sur des échantillons après une période de reconsolidation aux mêmes pressions que celles qu'ils avaient subies auparavant dans la cellule de consolidation. On a trouvé que ces deux types d'éva. luation de $\mathrm{c}_{\mathrm{u}}$ (essais au cône suédois et de cisaillement simple à volume constant) donnent des valeurs assez proches. Par ailleurs, les essais de mesure de $c_{\text {i }}$ sur des échantillons remaniés et conservés à volume constant ont montré que les gains de résistance dus à la thixotropie pour ces trois argiles sont relativement importants, particulièrement pour l'argile de Broadback où une valeur de $c_{u}$ égale à $6,1 \mathrm{kPa}$ a été relevée après une durée de conservation de deux mois.

Par ailleurs, l'examen des courbes illustrant l'évolution de $c_{\mathrm{u}}$ en fonction de $\sigma_{\mathrm{v}}^{\prime}$ a permis de distinguer trois zones:

- une première zone (zone I) dans laquelle $c_{4}$ croît fortement en fonction de $\sigma^{\prime}$, se traduit par des rapports $c_{\mathrm{v}} / \sigma_{v}^{\prime}$ élevés. Les valeurs élevées de $c_{\mathrm{u}} / \sigma_{\mathrm{v}}$, obtenues dans cette zone I, qui prend fin à $\sigma^{\prime}$ égal à $30 \mathrm{kPa}$ pour l'argile de Broadback et à $15 \mathrm{kPa}$ pour les autres argiles (Saint-Léon et Saint-Marcel), ont conduit à décrire ces trois argiles comme des argiles "surconsolidées». Cette surconsolidation a été attribuée à une structuration due particulièrement à une grande compression initiale favorisée ici par de fortes teneurs en eau initiale;

- une deuxième zone (zone II) dans laquelle $c_{\text { coît }}$ très peu en fonction de $\sigma_{v}^{\prime}$. On a attribué ce comportement à la structuration qui s'est développée précédemment dans la zone I. Par ailleurs, l'étendue de cette phase, en terme de contrainte, est sensiblement la même pour les trois argiles étudiées et elle vaut environ $30 \mathrm{kPa}$;

- une troisième zone (zone III) dans laquelle $c_{\mathrm{u}}$ croît de façon proportionnelle à $\sigma^{\prime}$, se traduit par un rapport $\mathrm{c} / \sigma^{\prime}$ constant et de valeur analogue à celle correspondant généralement pour les argiles normalement consolidées. 
Enfin, cette étude montre qu'il est possible d'évaluer en laboratoire la résistance au cisaillement d'un dépôt d'argile en voie de formation en milieu marin. Les essais in situ sont très difficiles à réaliser et ils requièrent la mise en place d'infrastructures très coûteuses alors que la méthodologie proposée requiert seulement le prélèvement d'échantillons remaniés. Il a aussi été montré que la salinité de l'eau utilisée en laboratoire n'a pas d'influence sur la forme des profils de résistance pour des pressions externes de consolidation identiques. Ceci indique que le remaniement de l'échantillon en laboratoire ne détruit pas totalement la structuration acquise initialement par l'argile lors de la déposition. En effet, en remaniant une argile on ne touche pas à la structure intergranulaire (DELAGE et LEBIHAN, 1986) et on recompose la structure intergranulaire par la consolida. tion. On retrouve donc des profils de résistance au cisaillement analogues à ceux en chantier. Ceci montre que la résistance au cisaillement est définie par les structures de l'argile à l'échelle intergranulaire affectée par le remaniement, et à l'échelle des particules au sein d'un agrégat. Ces structures qui sont typiques des conditions de dépôt seraient ainsi mémorisées.

\section{REMERCIEMENTS}

Cette étude a été réalisée grâce à la participation du personnel du laboratoire de mécanique des sols de l'Université de Sherbrooke. Le financement de ces travaux a été assumé par des subventions du Conseil de recherches en sciences naturelles et en génie du $\mathrm{Ca}$ nada (CRSNG) et du Fonds pour la formation des chercheurs et l'aide à la recherche du Québec (FCAR).

\section{BIBLIOGRAPHIE}

BALLIVY G., POULIOT G. et LOISELLE A. (1971), Quelques caractéristiques géologiques et minéra. logiques des dépôts d'argile du Nord-Ouest du Québec. Canadian Journal of Earth Sciences, V. 8, $\mathrm{n}^{\circ} 12$, pp. 1525-1541.

BENMOKRANE B. (1983), Contribution à l'étude de la résistance et de la consolidation d'argiles remaniées. Thèse de Maîtrise (MScA), Université de Sherbrooke (Québec), Département de génie civil, $259 \mathrm{p}$.

BJERRUM L and SIMONS N.E. (1960), Comparison of Shear Strength Characteristics of Normally Consolidated Clays, Proc. Res. Conf. Shear Strength Cohesion Soils, ASCE, Boulder, Col., pp. 711-726.

BJERRUM L. (1967), Engineering Geology of Normally Consolidated Marine Clays as Related to the Settlements of Buildings. Géotechnique, V.17, $n^{\circ} 2$, pp. 83-119.
BROWN J.D. and RASHID M.A. (1975), Geotechnical Properties of Nearshore Sediments of Canso Strait, Nova Scotia. Canadian Geotechnical Journal, V. $12, n^{\circ} 44$, pp. 44.57.

BUREAU DE NORMALISATION DU QUÉBEC, Détermination de la limite de liquidité à l'aide du pénétromètre à cône suédois et de la limite de plasticité. BNQ 2501-092, Québec.

DELAGE P. et LEBIHAN J.P. (1986), Microstructure et compressibilité d'argiles molles sensibles de l'Est canadien. CR Acad. Sc. Paris, t. 303, Série II, n¹9, pp. 1697.1702.

GARNEAU R and LEBIHAN J.P. (1977), Estimation of some properties of Champlain clays with the swedish fall cone. Canadian Geotechnical Journal, V. $14, n^{\circ} 4$, pp. 571-581.

JEFFERIES M.G., RUFFEL J.P., CROOKS J.H.A. and HUGUES J.M.O. (1985), Some Aspects of the Behavior of Beaufort Sea Clays. Strength Testing of Marine Sediments, Laboratory and in situ Measurements. ASTM, STP 883, Editors Chaney/Demars, pp. 487-514.

LEFEBVRE G. (1981), Etude en laboratoire des argiles du site de Broadback, complexe NBR. Rapport GEO-81-04, Département de génie civil, Université de Sherbrooke (Québec), 120 p.

LEROUEIL S., TAVENAS F et LEBIHAN J.-P. (1983), Propriétés caractéristiques des argiles de l'Est du Canada. Revue canadienne de géotechnique, V. 20 , pp. 681.705.

LOCAT J. (1982), Etude de l'origine de la structuration des argiles sensibles. Thèse de Doctorat (Ph.D.), Université de Sherbrooke (Québec), Département de génie civil, $512 \mathrm{p}$.

LOCAT J. and LEFEBVRE G. (1983), The compressibility and sensitivity of an artificially sediment clay soil: The Grande-Baleine marine clay, Québec, Canada. Marine Geotechnology, V. 6, n¹, pp. 1. 28.

LOCAT J. and LEFEBVRE G. (1986), The Origin of Structuration of the Grande-Baleine Marine Sediments, Québec, Canada. Quarterly Journal of Engineering Geology, V.16, pp. 365-374.

MESRI G., ROKHSAR A. and BOHOR B.H. (1975), Composition and Compressibility of Typical Samples of Mexico City Clay. Geotechnique, V. 25, $\mathrm{n}^{\circ} 3$, pp. 527.554.

MORIN P. and DAWE C.R. (1986), Geotechnical Properties of Two Deep Sea Marine Soils from Labrador Sea Area. 3rd Canadian Conference on Marine Geotechnical Engineering, St. John's, Newfoundland, V. 1, pp. 117-137.

MORISSETTE L. (1983), Dégradation des talus de la région de Saint-Léon-Le-Grand. Thèse de Maîtrise (MScA), Université de Sherbrooke (Québec) Département de génie civil, $339 \mathrm{p}$.

SKEMPTON A.W. and NORTHEY R.D. (1952), The Sensitivity of Clays. Geotechnique, V. III, $n^{\circ} 1$. 